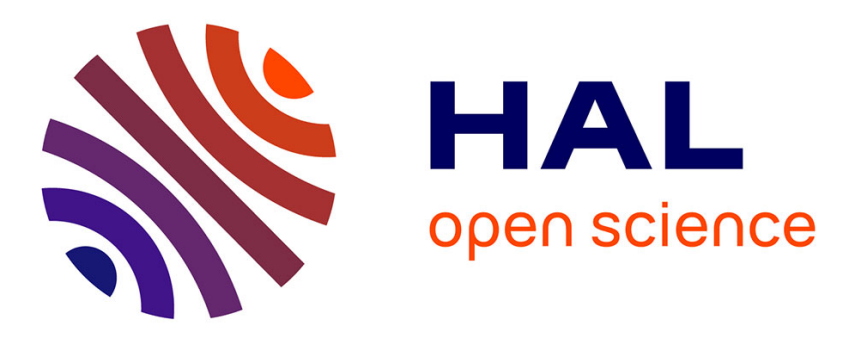

\title{
Channel bonding in cognitive radio wireless sensor networks
}

Husain Rehmani Mubashir, Stéphane Lohier, Abderrezak Rachedi

\section{To cite this version:}

Husain Rehmani Mubashir, Stéphane Lohier, Abderrezak Rachedi. Channel bonding in cognitive radio wireless sensor networks. ICOST'2012, Jul 2012, Avignon, France. pp.72 - 76, 10.1109/iCOST.2012.6271293 . hal-00690514

\section{HAL Id: hal-00690514 https://hal.science/hal-00690514}

Submitted on 8 Mar 2013

HAL is a multi-disciplinary open access archive for the deposit and dissemination of scientific research documents, whether they are published or not. The documents may come from teaching and research institutions in France or abroad, or from public or private research centers.
L'archive ouverte pluridisciplinaire HAL, est destinée au dépôt et à la diffusion de documents scientifiques de niveau recherche, publiés ou non, émanant des établissements d'enseignement et de recherche français ou étrangers, des laboratoires publics ou privés. 


\title{
Channel Bonding in Cognitive Radio Wireless Sensor Networks
}

\author{
Mubashir Husain Rehmani, Stéphane Lohier, and Abderrezak Rachedi \\ Université Paris-Est Marne-la-Vallée, LIGM, PasNet, France \\ E-mail: rehmani@univ-mlv.fr, lohier@univ-mlv.fr, rachedi@univ-mlv.fr
}

\begin{abstract}
Recently, wireless sensor networks are gaining a lot of attention due to the availability of various low cost sensor devices. For example, Wireless Multimedia Sensor Networks (WMSNs) have lot of new potential applications in different domains including, environmental monitoring, home automation, tracking, health care, just to name a few. Another example is the Body Area Networks (BANs), where different sensors are attached with the patient's body. However, these sensor networks also bring several challenges, such as they are resource constraint, requires application specific QoS requirements, high bandwidth demands, and resistance to interference. In this paper, we argue that wireless sensor nodes with cognitive radio capability, together with channel bonding, can help to address these challenges. In this perspective, we discuss cognitive radio wireless sensor network for hospital environment as a case study. We then discuss the way of spectrum characterization and criteria for channel bonding in such networks. Finally, we highlight issues and challenges, which provides the basis to develop algorithms and protocols for the future cognitive radio wireless sensor network deployments in different application scenarios.
\end{abstract}

Index Terms-Cognitive radio wireless sensor networks, wireless multimedia sensor networks, adjacent-channel interference, channel bonding.

\section{INTRODUCTION}

Wireless Multimedia Sensor Network (WMSN) [1] is an emerging technology and gaining a lot of attention by the researchers and industry. This is due to the availability of low cost sensor devices. Moreover, WMSN have a lot of new potential applications, such as, environmental monitoring, home automation, tracking, surveillance, and hospital environment applications [2]. However, there are several challenges in the practicability of WMSN and their performance is suffered by resource constrained wireless sensor nodes.. For instance, wireless multimedia sensor nodes require application specific QoS requirements, and high bandwidth demands. Another example is of Wireless Body Area Networks (BANs), which are prone to interference (cf. Section II).

In this paper, we argue that wireless sensor nodes with cognitive radio capability, i.e., Cognitive Radio Wireless Sensor Networks (CRWSNs) [3] can help to address these challenges. Additionally, channel bonding can further satisfy the bandwidth requirements of sensor nodes. In fact, in channel bonding, a set of contiguous non-overlapping channels are bonded

This work is part of the project entitled "Wireless Sensor Networks for vehicle and pedestrian traffic assessment applications", funded by the university of Paris Est under the PPS (Projet Pluriannuel Structurant) program. together to create a single bonded broadband channel. This results in large aggregated bandwidth, increase in the packet transmission rate, and the better satisfaction of the nodes bandwidth requirements [4]. Note that channel bonding [5] and channel aggregation [6] are two different concepts. In channel aggregation, aggregated channels need not to be contiguous as in the case of channel bonding. Channel aggregation need higher complexity and cost because of sophisticated channel management, scheduling, and load balancing.

In traditional wireless networks, channel bonding has been used for load balancing [4] and QoS provisioning [7]. The current draft version of IEEE 802.11n also discusses channel bonding in both the $2.4 \mathrm{GHz}$ and $5 \mathrm{GHz}$ spectrum, where two $20 \mathrm{MHz}$ channels are bonded into one $40 \mathrm{MHz}$ channel to improve transmission rates [8]. However, aforementioned techniques can not be directly applied to CRWSNs. This is due to the constraints imposed by Primary Radio (PR) nodes such as the time varying PR occupancy [9]. Moreover, for successful reception of data packets, the CRWS sender and receiver nodes are required to bond the same frequency channels. Thus, intelligent channel bonding techniques are required in CRWSNs that keeps into account the PR occupancy, cause less interference to PR nodes, and tune the CRWS sender/receiver nodes to the same bonded channels.

In this paper, our goal is to provide a clear picture of the potentials of channel bonding in cognitive radio wireless sensor networks. Furthermore, we also want to discuss how to minimize interference to PR nodes and satisfy the wireless sensor nodes' bandwidth requirement by equipping the wireless sensor nodes with cognitive radio capability. In this perspective, we first consider a cognitive radio wireless sensor network for hospital environment as a case study. Second, we describe the architecture and functionality of cognitive radio wireless sensor network in hospital environment. Third, we discuss the way of spectrum characterization and criteria for channel bonding in CRWSNs. And finally, we highlight issues and challenges, which provides the basis to develop algorithms and protocols for the future cognitive radio wireless sensor network deployments in different application scenarios.

The remainder of this paper is organized as follows: Section II discusses the CRWSN for hospital environment as a case study. In section III, we discuss channel bonding characterization. Issues and challenges are discussed in section IV, and finally, section $\mathrm{V}$ concludes the paper. 


\section{Cognitive Radio Wireless Sensor Network for Hospital EnVironment: A CASE Study}

In hospital environment, different e-health applications have been proposed to deliver patient's data, such as blood pressure, ECG, temperature, heart rate etc., to the clinical staff. This data is captured by the wireless sensor devices attached with the body of the patient, also called Body Area Networks (BANs) [10]. With the help of this data, doctors will be able to remotely monitor the patient and it will also helpful in telemedicine services [11], [12]. Normally, the data transfer from the patients' body to the monitoring room is done through wireless system such as ZigBee.

However, there are two main challenges. First, several overlapping wireless systems exists in the hospital environment besides ZigBee, for e.g., $802.11 \mathrm{~b} / \mathrm{g} / \mathrm{n}$, telemetry (which operate on WMTS bands or ISM bands), and Bluetooth [13]. All these wireless systems operates on the same band as ZigBee i.e., $2.4 \mathrm{GHz}$ ISM band and overlap with each other. This leads to harmful interference between the existing wireless systems.

More precisely, in cognitive radio wireless sensor networks, when $\mathrm{CR}$ wireless sensor nodes transmit on channels that are adjacent to the primary radio bands, it cause harmful interference to PR nodes [14]. For this reason, during channel bonding, the information regarding the adjacent channels' occupancy is crucial in mitigating interference to its adjacent primary radio nodes [15]. This problem is referred as Adjacent Channel Interference (ACI) problem [16], [17] in traditional wireless networks. Keeping this in mind, the Federal Communication Commission (FCC) in IEEE 802.22 standard has also restricted fixed devices from transmitting on adjacent channels of the $i^{\text {th }}$ active channel. Furthermore, in cognitive radio wireless sensor networks, the first priority is to protect the PR nodes, therefore, non-overlapping channels will be used for communication. In summary, it is essential, but extremely challenging to consider adjacent channel interference during channel bonding because of time varying PR occupancy.

Second, wireless communication by wireless sensor devices to the monitoring room may cause harmful interference to medical devices, which are sensitive to electromagnetic interference (EMI) caused by the wireless transmissions. Consequently, the medical devices may start malfunctioning, which cannot be tolerated due to the sensitivity of medical environment.

Recently, cognitive radio based architecture has been proposed to deal with the EMI problem in hospital environment [11], [12]. However, in this architecture, additional cognitive radio devices are required besides wireless sensor nodes. Moreover, different sensors may have different bandwidth requirements according to the application. For example, ECG data provided by the sensors may have high priority over temperature sensors. In addition, previous approaches [18], [19] [20] did not consider the adjacent channel interference problem while performing channel bonding.

Thus, to deal with these issues, we extend the idea of [11], [12] and propose a Cognitive Radio Wireless Sensor

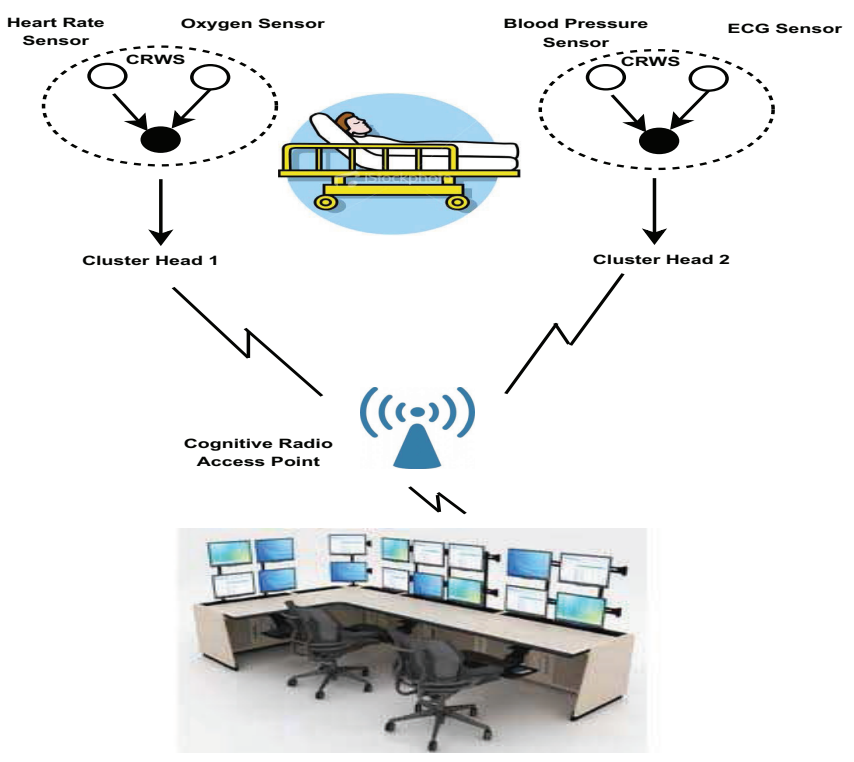

Fig. 1. Cognitive Radio Wireless Sensor Network in Hospital Environment

Networks (CRWSNs) [3] architecture (cf. Fig. 1) for hospital environment. In addition, we propose that the channel assignment should be done in such a manner that it should consider the PR occupancy on the adjacent channels.

We now describe the system model and assumptions.

\section{A. System Model and Assumptions}

We assume single-hop cognitive radio wireless sensor network architecture. In this architecture, cognitive radio access point (AP) is responsible for continuous spectrum monitoring of the PR nodes. Moreover, we assume that the AP is not resource constrained and is equipped with multiple transceivers. The AP performs channel characterization, channel assignment, and communicate the channel switching decision to CRWS nodes periodically.

We consider total $N$ channels in the network. We assume that the multiple sub-channels can be combined to create a single bonded channel (cf. Section III). We assume that cognitive radio wireless sensor nodes are equipped with a single transceiver, where a single channel can be assigned by the access point. This single channel is used for transmission or reception by the cognitive radio wireless sensor nodes. Both, the cognitive radio access point and cognitive radio wireless sensor nodes can freely switch to channels. We further assume that CRWS nodes may have different bandwidth requirements depending upon the application types.

\section{B. Architecture}

Cognitive Radio Wireless Sensor Network for Hospital Environment is a four-tier architecture tailored to implement and deploy real cognitive radio wireless sensor network applications in hospital environments. A general overview of our framework is depicted in Fig. 1. The building blocks of this architecture are: (1) Cognitive Radio Wireless Sensor (CRWS) nodes, (2) Cluster Heads (CHs), Cognitive Radio Access Point (AP), and (4) Medical Devices. 
In this architecture, we consider medical devices in the hospital as primary network and their nodes as primary nodes. Indeed, our objective is to detect on-going communications of the medical devices in order to protect them and then satisfy the bandwidth requirements of cognitive radio wireless sensor nodes. In our proposed architecture, the medical devices are considered as the Primary Radio (PR) nodes. These PR nodes have the higher priority to use the channels. In addition, when any PR transmission is going-on, all the other transmissions will be halted. We consider that each wireless sensor node is equipped with cognitive radio capability, which we refer hereafter as Cognitive Radio Wireless Sensor (CRWS) nodes [3]. In order to deal with bandwidth-hungriness problem of CRWS nodes, we propose to use channel boding.

We now describe individually the functionality of components:

\section{Functionality}

- Cognitive Radio Wireless Sensor (CRWS) Nodes: They are based on software defined radio. These nodes can access the AP for channel assignment. They can also create multi-hop network to reach nearest access point.

- Cluster Heads (CHs): They are cognitive radio wireless sensor nodes and their responsibility is to coordinate between cognitive radio access point and cognitive radio wireless sensor nodes. Cluster heads will help to create multi-hop network of CRWS nodes. Moreover, the election of cluster head can be done locally by using any appropriate cluster head technique [21].

- Cognitive Radio Access Point (AP): These devices are responsible for monitoring the spectrum usage by the primary radio devices. Moreover, they are also responsible for channel assignment to cognitive radio wireless sensor nodes based upon their bandwidth requirements.

- Medical Devices: These devices can be Oscilloscope, ECG etc. They operate on the ISM band and have higher priority to use the channels.

We now describe channel bonding characterization.

\section{Channel Bonding Characterization}

The main responsibility of AP is spectrum characterization. During spectrum characterization, the AP classifies the $N$ channels into occupied and unoccupied ones. The AP then characterizes the unoccupied channels into 2 major subcategories: (1) interior free channels and (2) border free channels. These two sub-categories are on the basis of the location of the $i^{\text {th }}$ free channel [15]:

- Interior Free Channel Case: In this case, the $i^{\text {th }}$ free channel is any interior free channel, i.e. $i \neq 1$ and $i \neq N$. This results in four different types of channels based upon free adjacent channels, i.e. Type I-1, I-2, I-3, and I-4 (cf. Fig. 2).

- Border Free Channel Case: In this case, the $i^{\text {th }}$ free channel is not any interior free channel and is located at the border of the spectrum, i.e. $i=1$ or $i=N$. This

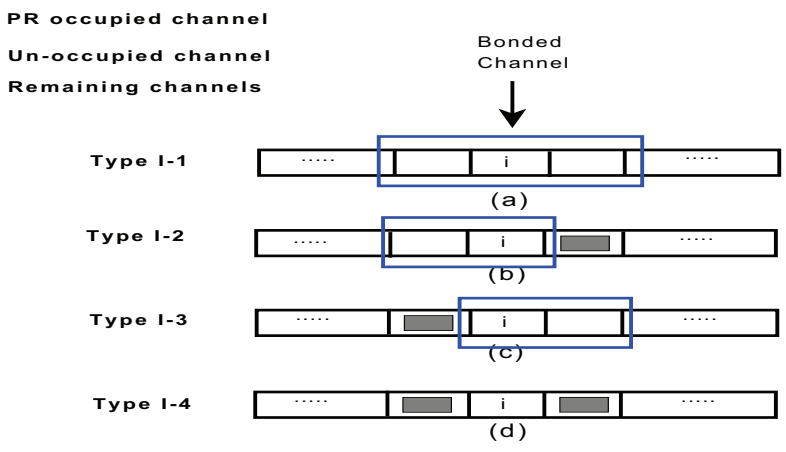

Fig. 2. Types of channels (Interior Free Channel Case): (a) Type I-1, higher bandwidth category, (b) Type I-2, higher bandwidth category, (c) Type I-3, higher bandwidth category, and (d) Type I-4, lower bandwidth category.

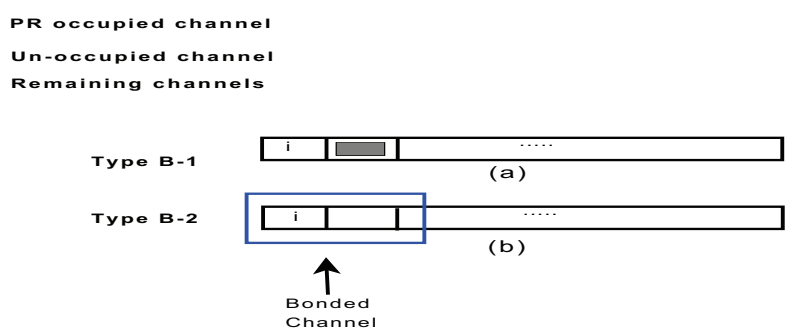

Fig. 3. Types of channels (Border Free Channel Case): (a) Type B-1, lower bandwidth category, and (b) Type B-2, higher bandwidth category.

results in two different types of channels based upon free adjacent channels, i.e. Type B-1, and B-2 (cf. Fig. 3).

We divide these six channel types into two sub-categories: (1) bonded channels category and (2) non-bonded channels category. In the bonded channels category, we propose to use channel Type I-1, I-2, I-3 and B-2. While, Type I-4 and B-1 will be treated as non-bonded channels.

After spectrum characterization, the AP will preform the spectrum assignment. The AP assigns bonded channels i.e. Type I-1, I-2, I-3, and B-2 channels to CRWS nodes that has higher bandwidth requirements, while Type I-4 and Type B1 channels will be assigned to CRWS nodes that has lower bandwidth requirements.

We now define the criteria for channel bonding, which is based on the availability of the number of free adjacent channels. This criterion will ensure the AP to mitigate the adjacent channel interference during channel bonding. Type I-1 channel has no adjacent neighbor channels occupied and results in three available contiguous non-overlapping channels. In Type I-1 channel case, the three channels will be bonded to create a single broadband channel. In Type I-2, I-3 and B-2 channel case, two contiguous non-overlapping channels are available. Therefore, these two channels will be bonded to create a single broadband channel. Finally, Type I-4 and Type B-1 channels have their adjacent channels occupied. Thus, in this case, no channel will be bonded and no transmission will be taken place.

We consider the channels of homogeneous bandwidth. A channel $i$ with center frequency $F_{c(i)}$ is shown in Fig. 4(a). 
The bandwidth $B W_{i}$ of channel $i$ can be written as:

$$
B W_{i}=F_{e(i)}-F_{s(i)}
$$

where $F_{s(i)}, F_{e(i)}$ are the starting and ending frequencies of channel $i$. The bandwidth of the channel $i$ from the starting or ending frequency to the center frequency $F_{c(i)}$ can be calculated as:

$$
B W_{c(i)}=F_{e(i)}-F_{c(i)}=F_{c(i)}-F_{s(i)}
$$

where $F_{s(i)}, F_{e(i)}$, and $F_{c(i)}$ are the starting, ending and center frequencies of channel $i$. We now take the case when three channels are combined to create a single bonded channel i.e. Type I channel. The bandwidth of bonded channel $i$ $B W_{B C(i)}^{3}$ (cf. Fig. 4(b)) that bonds the two adjacent channels (i-1) and (i+1) can be calculated as:

$$
\begin{array}{r}
B W_{B C(i)}^{3}=F_{e(i-1)}-F_{c(i-1)}+G B_{(i-1) \rightarrow i}+F_{e(i)} \\
-F_{s(i)}+G B_{(i) \rightarrow(i+1)}+F_{c(i+1)}-F_{s(i+1)}
\end{array}
$$

where (i-1) is the prior channel and (i+1) is the posterior channel of channel $i$. The symbols $F_{s(i)}, F_{e(i)}$, and $F_{c(i)}$ are the starting, ending and center frequencies. $G B_{(i-1) \rightarrow i}$ is the guard band between channel (i-1) and $i$ and $G B_{(i) \rightarrow(i+1)}$ is the guard band between channel $i$ and channel (i+1).

In order to avoid adjacent channel interference, we adopt an straightforward approach, in which the bandwidth of the bonded channel is increased till the center frequencies of adjacent channel $(i-1)$ and $(i+1)$ (cf. Fig. 4(b)) and the bandwidth is expressed in Eq. 3. We adopted this approach primarily because a large guard band is required at the band edges when performing channel bonding [5]. It is worth mentioning here that the guard band in between channels can be reused through channel bonding.

\section{Issues AND ChALLENGes}

In this section, we discuss the issues and challenges related with channel bonding in CRWSNs:

- How to conserve the energy of CRWS nodes? CRWS nodes are energy constrained devices. One challenge is to investigate the impact of channel bonding on the energy of CRWS nodes. More precisely, the higher the number of channels to be bonded, the higher the energy consumption, thus, there is a need to quantify this relationship of energy consumption vs. number of channels to be bonded.

- How to use channel for bursty traffic? Different types of CRWS nodes may generate bursty traffic. Thus, it is important to assign channel opportunistically to CRWS nodes to increase reliability and to decrease packet losses.

- How often the channel assignment is triggered? In traditional wireless networks, the channel assignment is often triggered when a new node join/leave the AP or a new data flow start by a node. In the context of CRWSNs, the appearance of PR nodes on the assigned channel may lead to trigger the channel assignment again. The channel assignment will become more frequent in the case of channel bonding, as the probability of PR appearing

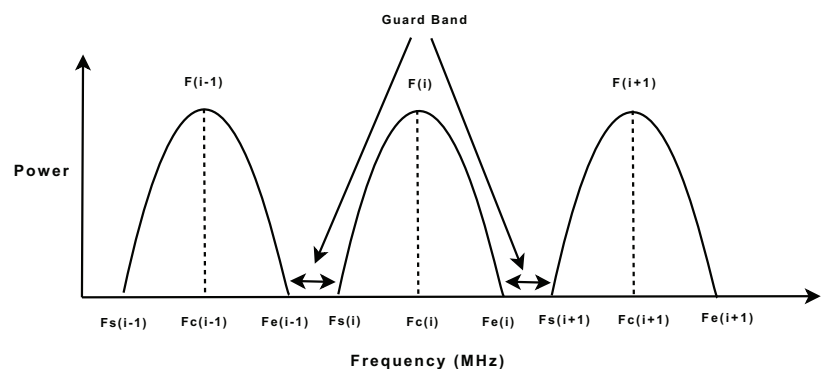

(a)

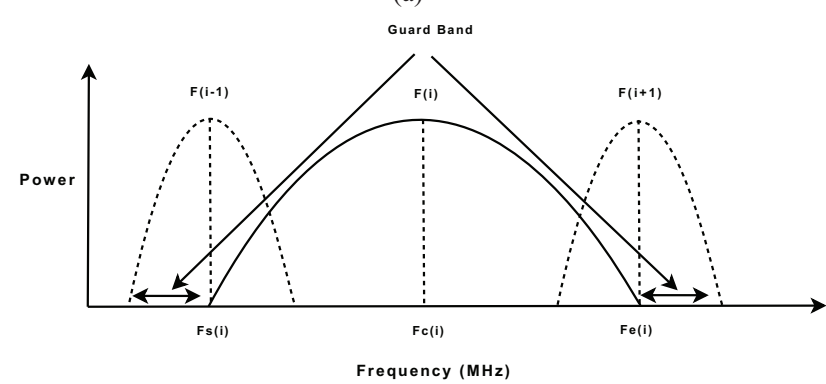

(b)

Fig. 4. An example showing channel bonding (a) when the channels are not bonded and (b) when three channels are bonded to create a single broadband bonded channel.

nodes increase with the number of used channels during channel bonding. The triggering of channel assignment can be minimized by predicting the PR activity.

- How to select stable free channels during channel bonding? PR nodes can re-appear on the bonded channel. Consequently, the bonded channel is broken and given back to PR nodes for transmission. The question is, how the transmission should be handed over seamlessly to another bonded channel with the same bandwidth size. One approach to deal with this problem is to maintain a pool of bonded channels at the AP. This pool contains the list of most probable channels for channel bonding w.r.t. their sizes. E.g., when a bonded channel with 2 sub-channels is broken then the AP select and assigns the next bonded channel of the same size to the CRWS node.

Note, if the pool of the bonded channels is already being used by the neighboring CRWS nodes and no free bonded channel is available, then CRWS node may use the nonbonded available channel for communication. However, this will reduce the data rate of the CRWS node.

- How to achieve maximum capacity gain while causing less interference to $P R$ nodes? We suggested to increase the bandwidth till the center frequency of the adjacent channels. Although, this ensures significant reduction in PR interference but there would be some capacity losses. In order to achieve maximum capacity gain while causing less interference to PR nodes, we have to consider the tradeoff between the channel separation distance and $P R$ interference. If the channel separation distance is small, PR may get interference, while if it is larger, capacity loss will be there. Thus, we need to quantify this tradeoff. 
- How many channels can be used for Bonding? One may think to bond as many channels for transmission but there exists a tradeoff; the higher the number of bonded channels, the higher is the probability of being interrupted by PR transmissions. Thus, one approach is to consider channels with longest remaining idle time for channel bonding.

- Which type of channels to be used for channel bonding? In CRWSNs, the available channels can be categorized into three types: (1) Overlapping, (2) Non-overlapping, and (3) Partially overlapping. The first priority in CRWSNs is to protect the PR nodes, therefore, overlapping and partially overlapping channels cannot be used for channel bonding. The only candidate for channel bonding in CRWSNs is non-overlapping channels (case of ZigBee Technology). However, the challenge resides in finding the consecutive non-overlapping idle channels.

- How interference can be handled? In CRWSNs, interference can be caused between: (1) CRWS-CRWS nodes, (2) CRWS-PR nodes, and (3) AP-AP. The interference between CRWS nodes can be caused when the AP assigned the channel to the CRWS node whose $i^{\text {th }}$ active channel is already being used by another CRWS node. The CRWS-PR interference can be caused when a channel is assigned to $\mathrm{CR}$ node whose $i^{t h}$ active channel is already being used by PR node. While, interAP interference can be caused if the same channel is assigned to the neighboring AP. All these three types of interference can be handled by employing an intelligent channel assignment on the AP. For instance, one way to reduce the CRWS-PR interference by the AP is to select those channels that have longest remaining idle time.

- Impact of channel bonding on Transmission Range and Throughput? When two or more channels are bonded together, the transmission range will vary and it will also impact the throughput of the wireless nodes. Thus, there is a need to investigate this issue also.

- How to deal with CRWS nodes occupancy? During channel assignment, besides PR occupancy, the AP also requires to consider the CRWS nodes occupancy on the adjacent channels. Otherwise, the CRWS nodes cause harmful interference among each other, which may degrade the performance of CRWS nodes. In order to deal with CRWS occupancy, the AP can assign those channels to CRWS nodes which are non-consecutive.

\section{CONCLUSION AND FUTURE WORK}

In this paper, we have discussed how wireless sensor nodes with cognitive radio capability, in conjunction with channel bonding, can satisfy the cognitive radio wireless sensor nodes' bandwidth requirements and interference problem. In this context, we took cognitive radio wireless sensor network for hospital environment as a case study. We have identified the working principle and the challenges related with the deployment of this architecture. Then, we discussed how the PR occupancy and adjacent-channel interference can be considered. We have also highlighted issues and challenges in the deployment of this architecture. We believe that this work can serve as a basis to build new algorithms and protocols for cognitive radio wireless sensor networks that can work in the presence of heterogeneous networks and communications technologies in different application environments.

\section{ACKNOWLEDGEMENTS}

The author, Mubashir Husain Rehmani, would like to thank Aline Carneiro Viana from INRIA, France for the helpful discussions and suggestions.

\section{REFERENCES}

[1] I. F. Akyildiz, T. Melodia, and K. R. Chowdhury, "A survey on wireless multimedia sensor networks," Comput. Netw., vol. 51, pp. 921-960, March 2007.

[2] - "Wireless multimedia sensor networks: Applications and testbeds," Proceedings of the IEEE, vol. 96, No. 10, October 2008.

[3] O. B. Akan, O. B. Karli, and O. Ergul, "Cognitive radio sensor networks," IEEE Network, vol. 23, issue 4, pp. 34-40, 2009.

[4] R. Chandra, R. Mahajan, T. Moscibroda, R. Raghavendra, and P. Bahl, "A case for adapting channel width in wireless networks," SIGCOMM Comput. Commun. Rev., vol. 38, pp. 135-146, August 2008.

[5] IEEE P202.22 Draft Standard for Wireless Regional Area Networks : Channel Bonding versus Channel Aggregation.

[6] IEEE P202.22 Draft Standard for Wireless Regional Area Networks Channel Aggregation Summary.

[7] V. Raman and M. Caesar, "A practical approach for providing qos in multichannel ad-hoc networks using spectrum width adaptation," in IEEE GLOBECOM 2009, 30 2009-dec. 4 2009, pp. 1 -6.

[8] IEEE 802.11n draft. [Online]. Available: http://www.ieee802.org/11/ Reports/tgn_update.htm

[9] I. F. Akyildiz, W.-Y. Lee, M. C. Vuran, and S. Mohanty, "Next generation/dynamic spectrum access/cognitive radio wireless networks: a survey," Computer Network Journal, vol. 50 , Issue 13, 2006.

[10] B. Latré, B. Braem, I. Moerman, C. Blondia, and P. Demeester, "A survey on wireless body area networks," Wirel. Netw., vol. 17, pp. 1-18, January 2011

[11] P. Phunchongharn, E. Hosssain, D. Niyato, and S. Camorlinga, "A cognitive radio system for e-health applications in a hospital environment," IEEE Wireless Communications Magazine, pp. 20-28, Feburary 2010.

[12] S. Feng, Z. Liang, and D. Zhao, "Providing telemedicine services in an infrastructure-based cognitive radio network," IEEE Wireless Communications Magazine, pp. 96-103, Feburary 2010.

[13] C.-J. M. Liang, N. B. Priyantha, J. Liu, and A. Terzis, "Surviving wi-fi interference in low power zigbee networks," in 8th ACM SenSys, ser. SenSys '10. New York, NY, USA: ACM, 2010, pp. 309-322.

[14] H. Tang, "Some physical layer issues of wide-band cognitive radio systems," in IEEE DySPAN 2005, nov. 2005, pp. $151-159$.

[15] M. B. R. C. Ghosh, S. Roy and D. P. Agrawal., "Spectrum occupancy modeling and validation using real-time measurements," in ACM CoRoNet'10 in conjunction with MobiCom 2010, September 2010.

[16] V. Raman and N. H. Vaidya, "Adjacent channel interference reduction in multichannel wireless networks using intelligent channel allocation," Technical report, University of Illinois at Urbana-Champaign, August, Tech. Rep., 2009.

[17] A. S. S. Mishra and R. Brodersen, "Cooperative sensing among cognitive radios," in IEEE ICC, 2006.

[18] H. Rahul, N. Kushman, D. Katabi, C. Sodini, and F. Edalat, "Learning to share: Narrowband-friendly wideband wireless networks," in $A C M$ SIGCOMM, vol. 38 , Issue 4, 17-22 AUGUST 2008, pp. 147-158.

[19] G. Yuan, R. Grammenos, Y. Yang, and W. Wang, "Performance analysis of selective opportunistic spectrum access with traffic prediction," Vehicular Technology, IEEE Transactions on, vol. 59, no. 4, pp. 1949 -1959 , may 2010.

[20] L. Jiao, V. Pla, and F. Li., "Analysis on channel bonding/aggregation for multi-channel cognitive radio networks," in European Wireless Conference (EW), 2010.

[21] A. A. Abbasi and M. Younis, "A survey on clustering algorithms for wireless sensor networks," Computer Communications, vol. Volume 30 Issue 14-15, October, 2007. 